\title{
Protein kinase $C$ isoforms $\alpha, \delta$ and $\theta$ require insulin receptor substrate-1 to inhibit the tyrosine kinase activity of the insulin receptor in human kidney embryonic cells (HEK 293 cells)
}

\author{
M. Kellerer ${ }^{1}$, J. Mushack ${ }^{1}$, E. Seffer ${ }^{1}$, H. Mischak ${ }^{2}$, A. Ullrich ${ }^{3}$, H. U. Häring ${ }^{1}$ \\ ${ }^{1}$ Eberhard-Karls-Universität Tübingen, Innere Medizin IV, Tübingen, Germany \\ ${ }^{2}$ Hämatologisches Institut, München, Germany \\ ${ }^{3}$ Max-Planck-Institut für Biochemie, Martinsried, Germany
}

\begin{abstract}
Summary Protein kinase $\mathrm{C}(\mathrm{PKC})$ isoforms are potentially important as modulators of the insulin signalling chain and could be involved in the pathogenesis of cellular insulin resistance. We have previously shown that phorbol ester stimulated PKC $\beta 1$ and $\beta 2$ as well as tumor necrosis factor- $\alpha(\mathrm{TNF} \alpha)$ stimulated $\mathrm{PKC} \varepsilon$ inhibit human insulin receptor (HIR) signalling. There is increasing evidence that the insulin receptor substrate-1 (IRS-1) is involved in inhibitory signals in insulin receptor function. The aim of the present study was to elucidate the role of IRS- 1 in the inhibitory effects of protein kinase $\mathrm{C}$ on human insulin receptor function. HIR, PKC isoforms $(\alpha, \beta 1$, $\beta 2, \gamma, \delta, \varepsilon, \eta, \theta$ and $\zeta)$ and IRS- 1 were coexpressed in human embryonic kidney (HEK) 293 cells. PKCs were activated by preincubation with the phorbol ester 12-O-tetradecanoylphorbol 13-acetate (CTPA) $\left(10^{-7} \mathrm{~mol} / \mathrm{l}\right)$ following insulin stimulation. While PKCs $\alpha, \delta$ and $\theta$ were not inhibitory in HEK 293 cells which were transfected only with HIR and PKC, additional transfection of IRS-1 induced a strong inhibitory effect of these PKC isoforms being maximal for
\end{abstract}

PKC $\theta(99 \pm 1.8 \%$ inhibition of insulin stimulated receptor autophosphorylation, $n=7, p<0.001)$. No effect was seen with PKC $\gamma, \varepsilon, \zeta$ and $\eta$ while the earlier observed insulin receptor kinase inhibition of PKC $\beta 2$ was further augmented $(91 \pm 13 \%, \quad n=7$, $p<0.001$ instead of $45 \%$ without IRS-1). The strong inhibitory effect of PKC $\theta$ is accompanied by a molecular weight shift of IRS-1 (183 kDa vs $180 \mathrm{kDa})$ in the sodium dodecyl sulphate polyacrylamide gel. This can be reversed by alkaline phosphatase treatment of IRS-1 suggesting that this molecular weight shift is due to an increased phosphorylation of IRS-1 on serine or threonine residues. In summary, these data show that IRS-1 is involved in the inhibitory effect of the PKC isoforms $\alpha, \beta 2, \delta$ and $\theta$ and it is likely that this involves serine/threonine phosphorylation of IRS-1. [Diabetologia (1998) 41: 833-838]

Keywords Insulin receptor, insulin receptor substrate, protein kinase $\mathrm{C}$, insulin resistance, serine phosphorylation.
Insulin resistance of target tissues plays a major role in the pathogenesis of Type II (non-insulin-dependent) diabetes mellitus. In particular insulin resis-

Received: 11 February 1998 and in revised form 2 April 1998

Corresponding author: Dr. M. Kellerer, Eberhard-Karls-Universität Tübingen, Innere Medizin IV, Otfried-Müller-Strasse 10, D-72076 Tübingen, Germany

Abbreviations: TPA, 12-O-Tetradecanoylphorbol 13-actetate; HIR, human insulin receptor; IRS, insulin receptor substrate; PKC, protein kinase C; HEK, human embryonic kidney cells; $\mathrm{PMSF}$, phenylmethylsulphonyl fluoride; $\mathrm{TNF} \alpha$, tumor necrosis factor $\alpha$; FCS, fetal calf serum. tance of the skeletal muscle appears to be of quantitative importance in the early stages of the development of the disease [1]. The molecular mechanisms of skeletal muscle insulin resistance are still not understood. On the cellular level insulin resistance may be caused at multiple steps of the insulin signalling pathway. Among the most interesting candidates which can inhibit insulin signalling are tyrosine phosphatases and serine kinases. The serine/threonine kinase protein kinase $\mathrm{C}(\mathrm{PKC})$ is of predominant interest as a potential inhibitor of insulin signalling function [2]. Different PKC isoforms represent a family of structurally and functionally related proteins 
which are derived from multiple genes as well as from alternative splicing of single mRNA transcripts [3,4]. $\mathrm{PKC}$ isoforms differ in their regulatory domains, in their dependence on calcium as well as in their tissue distribution [3,4]. They are subdivided into three major groups: classical calcium dependent cPKC isoforms $\alpha, \beta 1, \beta 2, \gamma$; new calcium independent $\mathrm{nPKC}$ isoforms $\delta, \varepsilon, \eta, \theta$; and atypical aPKC isoforms $\xi, \lambda$ and $\mu$ which cannot be activated by phorbol esters.

We have earlier characterized the mechanism of hyperglycaemia induced insulin receptor inhibition in different cell types [5]. According to these studies a glucose induced activation of protein kinase $\mathrm{C}$ appears to be involved in the hyperglycaemia dependent insulin resistance [5]. Furthermore, we found evidence that PKC $\varepsilon$ might also contribute to the inhibitory effect of tumor necrosis factor $\alpha(\mathrm{TNF} \alpha)$ on insulin signalling [6]. To investigate which PKC isoforms might be involved in inhibition of the insulin signalling chain we performed cotransfection studies with human insulin receptor and different PKC isoforms in human embryonic kidney cells (HEK 293 cells). HEK 293 cells are a cell system with low endogenous expression of insulin-signalling elements. Upon transfection with insulin receptor or other elements of the insulin signalling chain transiently high expression levels of the respective proteins are obtained. Even though this is an artificial cell system, it offers unique possibilities to study specifically the interaction of signalling elements of interest. In this system we found that the phorbol ester activated PKC isoforms $\beta 1$ and $\beta 2$ are able to cause a significant reduction of the insulin receptor autophosphorylation [2]. Now increasing evidence is being presented that the insulin receptor substrate-1 (IRS-1) can be involved in inhibitory effects on insulin signalling $[7,8$, 9]. To investigate the role of IRS- 1 in the interaction of human insulin receptor (HIR) and $\mathrm{PKC}$ isoforms experiments with HEK 293 cells transfected with HIR, PKC and IRS-1 were performed. We found that coexpression of IRS-1 allows other PKC isoforms than $\beta 1$ and $\beta 2$ to exert an inhibitory effect on HIR function. It is of particular interest that PKC $\theta$ has a strong inhibitory effect on HIR activity because this isoform is expressed predominantly in human skeletal muscle and it can be speculated that PKC $\theta$ is quantitatively important in the pathogenesis of skeletal muscle insulin resistance.

\section{Materials and methods}

Materials. Cell culture reagents and fetal calf serum (FCS) were purchased from Gibco (Eggenstein, Germany); culture dishes were from Greiner (Frickenhausen, Germany). Porcine insulin, aprotinin, phenylmethylsulphonyl fluoride (PMSF), 12-O-tetradecanoylphorbol (TPA), $\mathrm{Na}_{3} \mathrm{VO}_{4}$, Triton X-100, dithiothreitol (DTT), akaline phosphatase coupled to agarose beads (P0762) and p-nitrophenyl phosphate were from Sigma
(Munich, Germany). Reagents for SDS-PAGE and Western blotting were obtained from Roth (Karlsruhe, Germany) and Biorad (Munich, Germany) Qiagen plasmid kit was from Oiagen, Hilden, Germany. Nitrocellulose was from Schleicher \& Schuell (Dassel, Germany). Visualization of immunocomplexes after Western blotting was performed with the non-radioactive enhanced chemiluminescence system (ECL, Amersham Buchler, Braunschweig, Germany). Anti-pTyr antibodies (PY20) were from Leinco Technologies (Ballwin, USA). The insulin receptor antibody against the $\mathrm{C}$-terminal domain of the $\beta$-subunit ( $\alpha \mathrm{CT}-1)$ was kindly provided from $\mathrm{R}$. Lammers (Tuebingen, Germany). Polyclonal IRS-1 antibody was a gift from M.F. White (Boston, USA). Polyclonal isoform specific PKC antibodies and panPKC antibody were purchased from Gibco (Eggenstein, Germany). The c-DNA of the insulin receptor was from human origin, c-DNA from IRS was from rat, those of the PKC isoforms were from mouse origin.

Transient expression of HIR and PKC isoforms in HEK 293 cells. The cDNA for the wild-type insulin receptor, IRS-1 or the different PKC isoforms $(\alpha, \beta 1, \beta 2, \gamma, \delta, \varepsilon, \eta, \theta$ and $\zeta)$ were cloned into a cytomegalovirus promoter-based expression vector and plasmid DNA was prepared using a Qiagen Plasmid Kit. Human embryonic kidney (HEK) 293 cells (ATCC CRL 1573) were grown in Dulbecco's MEM/Nutrient Mix F12 medium supplemented with $10 \%$ FCS. A total of $4 \mu \mathrm{g}$ plasmid DNA was transfected per semiconfluent 35-mm diameter dish as described [2]. Cultures were maintained over night at $37^{\circ} \mathrm{C}, 3 \% \mathrm{CO}_{2}$. The medium was changed to Dulbecco's MEM/Nutrient Mix F12 medium without FCS containing $2 \mathrm{mmol} / \mathrm{l}$ glutamine $20 \mathrm{~h}$ before the experiment. Cells were then preincubated with $10^{-7} \mathrm{~mol} / \mathrm{l} \mathrm{TPA}$ for $15 \mathrm{~min}$ before stimulation with $10^{-7} \mathrm{~mol} / \mathrm{l}$ insulin $(3 \mathrm{~min})$. Subsequently, cells were lysed in $200 \mu \mathrm{l}$ lysis buffer $(50 \mu \mathrm{mol} / \mathrm{l}$ HEPES $\mathrm{pH} 7.2$, $150 \mathrm{mmol} / 1 \mathrm{NaCl}, 1 \mathrm{mmol} / 1$ EGTA, $10 \%$ (v/v) glycerol, $1 \%$ (v/ v) Triton $\mathrm{X}-100,100 \mathrm{mmol} / \mathrm{l} \mathrm{NaF}, 10 \mathrm{mmol} / \mathrm{l}$ sodium pyrophosphat, $100 \mu \mathrm{mol} / \mathrm{l}$ sodium orthovanadat, $1 \mathrm{mmol} / \mathrm{l} \mathrm{PMSF}, 10 \mu \mathrm{g} /$ $\mathrm{ml}$ aprotinin). The lysates were centrifuged for $10 \mathrm{~min}$ at $14000 \mathrm{~g}, 40 \mu \mathrm{l}$ of the supernatant was taken, $5 \times$ Laemmli buffer added, boiled for $5 \mathrm{~min}$, and separated by $7.5 \%$ SDSPAGE.

Alkaline phosphatase treatment of cell lysates. HEK 293 cells were transfected with HIR, PKC $\theta$ and IRS- 1 and stimulated with or without phorbol ester TPA $\left(10^{-7} \mathrm{~mol} / 1,15 \mathrm{~min}\right)$ prior to insulin stimulation $\left(10^{-7} \mathrm{~mol} / 1,3 \mathrm{~min}\right)$. Lysates from phorbol ester stimulated cells were either incubated with agarose beads alone or with alkaline phosphatase attached to agarose beads ( $\sim 800 \mathrm{U}$ alkaline phosphatase) in a buffer containing $50 \mathrm{mmol} / 1 \mathrm{HEPES}, 1 \mathrm{mmol} / \mathrm{l} \mathrm{PMSF}$ and $0.2 \%$ Triton X-100, $\mathrm{pH}$ 7.8. Samples were rotated for $2 \mathrm{~h}$ at $4{ }^{\circ} \mathrm{C}$ in this buffer together with agarose. The supernatant was separated from agarose beads by centrifugation and was boiled with $5 \times$ Laemmli buffer for $5 \mathrm{~min}$. 7.5\% SDS-polyacrylamide gel electrophoresis and Western blotting was performed. As a control p-nitrophenyl phosphate $(50 \mathrm{mmol} / \mathrm{l})$ was used as a substrate for alkaline phosphatase in the same conditions mentioned above (HEPES buffer, $\mathrm{pH} 7.8,4^{\circ} \mathrm{C}$ ). P-nitrophenyl phosphatase turned yellow after less than 3 min incubation at $4^{\circ} \mathrm{C}$ which indicates dephosphorylation of $\mathrm{p}$-nitrophenyl phosphate and confirms that alkaline phosphatase is able to dephosphorylate substrates under the conditions used for IRS-1 dephosphorylation.

Western blotting. After electrophoresis, proteins were transferred to nitrocellulose by electroblotting (transfer buffer: $20 \mathrm{mmol} / 1 \mathrm{NaH}_{2} \mathrm{PO}_{4}$ and $20 \mathrm{mmol} / \mathrm{N} \mathrm{Na}_{2} \mathrm{HPO}_{4}, \mathrm{pH} \mathrm{8.8)}$. After 
transfer, the nitrocelluloses were blocked with NET buffer $(150 \mathrm{mmol} / \mathrm{l} \mathrm{NaCl}, 5 \mathrm{mmol} / \mathrm{l}$ EDTA, $50 \mathrm{mmol} / \mathrm{l}$ Tris, $0.05 \%$ Triton $\mathrm{X}-100$ and $0.25 \%$ gelatine, $\mathrm{pH} 7.4$ ) for $1 \mathrm{~h}$. Subsequently, they were incubated with the first antibodies $(\alpha$ PY20, $\alpha$ CT -1 , $\alpha$ IRS- 1 or PKC antibodies in NET buffer) overnight at $4{ }^{\circ} \mathrm{C}$. The nitrocellulose membranes were washed $4 \times 10$ min with NET buffer before incubating with horseradish peroxidaseconjugated anti-rabbit IgG or anti-mouse $\mathrm{IgG}$ for $1 \mathrm{~h}$ at room temperature. Visualization of immunocomplexes was performed by enhanced chemiluminescence (ECL, Amersham). To remove antibodies before reblotting the nitrocellulose was incubated in $62.5 \mathrm{mmol} / \mathrm{l}$ Tris- $\mathrm{HCl}, \mathrm{pH} 6.8,2 \% \mathrm{SDS}$ and $100 \mathrm{mmol} / \mathrm{l} \beta$-mercaptoethanol for $30 \mathrm{~min}$ at $55^{\circ} \mathrm{C}$.

\section{Results}

HEK 293 cells were cotransfected with HIR, IRS-1 and different PKC isoforms $(\alpha, \beta 1, \beta 2, \gamma, \delta, \varepsilon, \eta, \theta$ and $\xi)$. Transfected 293 cells were incubated with TPA $\left(10^{-7} \mathrm{~mol} / \mathrm{l}\right)$ for $15 \mathrm{~min}$ to activate PKCs and then stimulated with insulin $\left(10^{-7} \mathrm{~mol} / \mathrm{l}\right)$ for $3 \mathrm{~min}$. Whole cell lysates were separated on a $7.5 \%$ SDSPAGE, blotted onto nitrocellulose and immunodetected with anti-phosphotyrosine, anti-insulin receptor (not shown), anti-IRS-1 and different PKC isoform antibodies. Figure 1 shows a representative autoradiogram. Immunoblots with IRS-1 antibodies indicate that similar amounts of protein are expressed. In addition the expression of insulin receptor was equal in all samples on the Western blot (not shown). Immunoblots with $\alpha \mathrm{PKC}$ antibodies demonstrate that all PKC isoforms are overexpressed (Fig.1). Due to different affinities of the PKC isoform specific antibodies a quantitative comparison of the expression level of the various isoforms is, however, not possible. Insulin stimulated tyrosine phosphorylation reaches a factor of $5.8 \pm 2.4$-fold $(n=7)$ autophosphorylation for endogenous IRS-1 although probably due to the already high basal level in cells cotransfected with HIR and IRS- 1 only a $1.8 \pm 0.47$-fold $(n=7)$ further increase of tyrosine phosphorylation is observed. This stimulatory effect is clearly below the stimulation factor observed for the insulin receptor autophosphorylation. Autophosphorylation for endogenous insulin receptor is not detectable. In contrast, parental HEK 293 cells transfected with HIR show at least a 10-fold stimulation of insulin receptor autophosphorylation. It is indicated from the antiphosphotyrosine immunoblot that the effect of insulin on receptor autophosphorylation is reduced in phorbolester treated cells cotransfected with HIR and IRS-1 ( $20 \pm 32 \%$ inhibition, $n=9, p<0.01)$, respectively cotransfected with HIR, IRS-1 and PKC $\alpha, \beta 1, \beta 2, \delta$, and $\theta$. No additional effect to endogenous PKC is seen in cells transfected with PKC $\gamma, \varepsilon$, $\eta$ and $\zeta$. Since PKC $\zeta$ is a phorbol ester independent aPKC-isoform we can only show in this case that overexpression itself does not induce inhibition of the insulin receptor autophosphorylation. Quantita- tive data from scanning densitometry in seven experiments are also included in Figure 1 by bars which indicate per cent of inhibition of the insulin stimulated receptor autophosphorylation by different PKC isoforms. The inhibitory effect on insulin receptor autophosphorylation is most pronounced in PKC $\beta 2$ and PKC $\theta$ transfected cells. Quantification by scanning densitometry showed an inhibition of insulin stimulated phosphorylation of $99 \pm 1.8 \% \quad(n=7$, $p<0.001)$ for PKC $\theta$ and $91 \pm 13 \%(n=7, p<0.001)$ for PKC $\beta 2$. In these cells tyrosine phosphorylation of IRS- 1 is also inhibited $(69 \pm 22 \%, n=7, p<0.001$ for PKC $\theta$ and $45 \pm 27 \%, n=7, p<0.01$ for $\mathrm{PKC} \beta 2$ ).

In cells transfected with PKC $\beta 2$ or PKC $\theta$ a $3 \mathrm{kDa}$ molecular weight shift of IRS-1 is evident from the immunoblot with anti-IRS- 1 antibodies. This molecular weight shift is also seen, however less pronounced, in cells cotransfected with PKC $\alpha, \beta 1$ and PKC $\delta$ and is possibly due to an increased phosphorylation of IRS-1. As tyrosine phosphorylation of IRS-1 is reduced at least in the cotransfection with PKC $\beta 2$ or $\operatorname{PKC} \theta$, it can be concluded that the increase in phosphorylation has to occur on serine or threonine residues. To test this hypothesis we removed phosphorus from IRS- 1 by alkaline phosphatase treatment. Figure 2 shows the result of this experiment. HEK 293 cells were cotransfected with HIR, IRS- 1 and PKC $\theta$ and incubated with or without phorbol ester TPA prior to insulin stimulation. One aliquot of the cell lysate was applied on $7.5 \%$ SDS-PAGE and blotted against anti-phosphotyrosine antibody to confirm inhibition of insulin receptor- and IRS-1 phosphorylation after phorbolester treatment. PKC $\theta$ expression was compared by reblotting the same nitrocellulose with PKC $\theta$ antibody. Another aliquot from the same experiment was used for incubation with or without alkaline phosphatase attached to agarose beads and subsequently Western blots were performed against IRS-1 antibodies (Fig. 2, upper pannel). Under the experimental conditions where an inhibition of tyrosine phosphorylation of HIR and IRS-1 is found (Fig. 2, ptyr immunoblot) the molecular weight shift is evident from the immunoblot against the IRS-1 antibodies. This molecular weight shift of $3 \mathrm{kDa}$ can be reversed by incubation of the cell lysate with alkaline phosphatase (Fig.2, aIRS immunoblot, upper pannel).

\section{Discussion}

In previous studies we have cotransfected insulin receptor and PKC isoforms only, but not IRS-1 to investigate $\mathrm{PKC}$ action on insulin signalling. In this situation only PKC $\beta 1$ and $\beta 2$ were clearly inhibitory on insulin receptor autophosphorylation [2]. PKC $\alpha$ showed a variable inhibitory effect which did not reach statistical significance while all other isoforms 

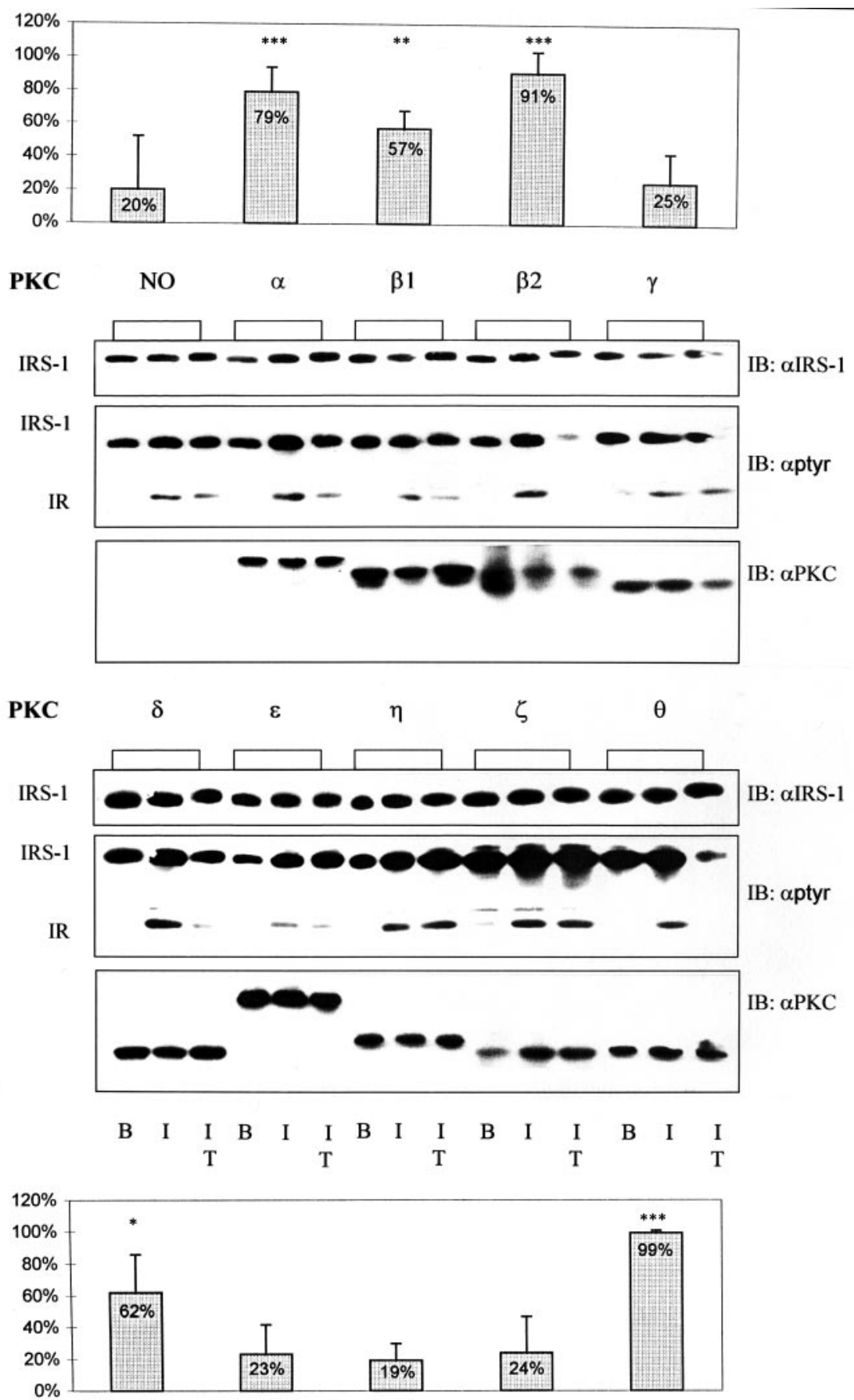

Fig. 1. Inhibition of insulin receptor $\beta$-subunit- and IRS-1phosphorylation. HEK 293 cells were transfected with cDNA of the human insulin receptor, IRS-1 and of different PKC isoforms as indicated. Transfected HEK 293 cells were incubated with $10^{-7} \mathrm{~mol} / \mathrm{l}$ TPA for $15 \mathrm{~min}$ prior to insulin stimulation $\left(10^{-7} \mathrm{~mol} / \mathrm{l}, 3 \mathrm{~min}\right)$. Whole cell lysates were separated on a $7.5 \%$ SDS PAGE, blotted onto nitrocellulose and immunodetected with anti-phosphotyrosine antibody $\alpha$ Ptyr (as indicated on the right side). The same nitrocellulose was then reprobed with anti-IRS- 1 antibody ( $\alpha$ IRS- 1 , indicated on the right side) and the appropriate anti-PKC antibodies $(\alpha \mathrm{PKC})$. Pan-PKC antibody was used for detection of $\operatorname{PKC} \alpha, \beta 1, \beta 2$ and $\gamma$. All other PKCs were detected by isoform specific antibodies. Quantitative analysis of seven experiments (\% inhibition of insulin stimulated insulin receptor autophosphorylation) are given in bars (mean \pm SD) at the top and at the bottom of the Western blot. In control experiments where only HIR and PKC $\theta$ are cotransfected no inhibitory effect on HIR autophosphorylation was observed (Westernblot not shown). Under these conditions insulin stimulated HIR-autophosphorylation was after TPA incubation $98 \pm 36 \%$ of the value seen after insulin stimulation alone. Statistical analysis was performed by Student's t-test for paired samples $(* p<0.05$, ** $p<0.01$, $* * * p<0.001)$ 

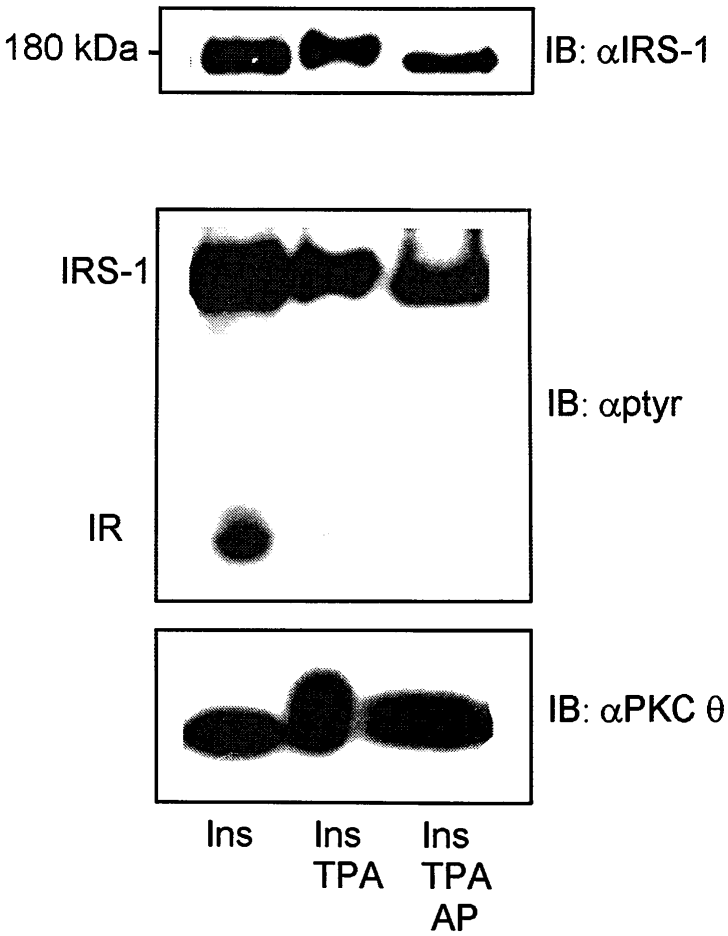

Fig. 2. Alkaline phosphatase treatment of cell lysates. HEK 293 cells overexpressing HIR, IRS- 1 and PKC $\theta$ were preincubated with or without the phorbol ester TPA prior to insulin stimulation (insulin $10^{-7} \mathrm{~mol} / \mathrm{l}, 3 \mathrm{~min}$ ). One aliquot of the cell lysate was directly applied to $7.5 \%$ SDS gel electrophoresis and the inhibition of insulin receptor- and IRS-1 phosphorylation was confirmed by a Western blot against an anti-ptyr antibody (indicated on the right side). Further, the expression level of PKC $\theta$ was compared between the samples in an immunoblot against PKC $\theta$ antibodies (lower pannel). Another aliquot of the cell lysate was incubated with alkaline phosphatase prior to gel electrophoresis and immunoblotting with anti-IRS-1 antibodies (upper pannel). After treatment with alkaline phosphatase the molecular weight shift of $3 \mathrm{kDa}$ is reversed which can be seen on the immunoblot against $\alpha$ IRS-1 (upper pannel)

were ineffective [2]. The present data now show that the level of IRS-1 expressed in these cells determines whether other PKC isoforms are as well inhibitory to insulin receptor activity. It is possible that the inhibitory effects observed in cells cotransfected with HIR and $\mathrm{PKC} \beta 1$ or $\beta 2$ occur independently of the presence of IRS-1. Alternatively it can be argued that the endogenous level of IRS-1 in HEK 293 cells is sufficient to allow inhibitory interaction with $\mathrm{PKC} \beta$. Cotransfection of HEK 293 cells with HIR, PKC and IRS-1 provides a system where all three signalling proteins are expressed in high amounts. This condition is obviously needed to allow an inhibitory interaction of other PKC isoforms besides $\beta 1$ and $\beta 2$. High level of tyrosine phosphorylation of IRS- 1 is already seen in the basal state. This can only be observed when IRS- 1 is coexpressed with HIR which suggests that the basal activity of HIR if present in high amounts is sufficient to phosphorylate IRS-1. But those conditions are still sufficient to demonstrate inhibition of IRS-1 phosphorylation by $\mathrm{PKC}$ which is shown with $\mathrm{PKC}$ isoforms $\beta 2$ and $\theta$ in our experiments.

The observation that PKC $\theta$ has an especially strong inhibitory effect on insulin receptor signalling is of particular interest since it was shown that PKC $\theta$ content and cellular distribution correlates with insulin sensitivity in insulin resistant animal models [10]. In addition, PKC $\theta$ is expressed predominantly in human skeletal muscle. This PKC isoform would therefore be an interesting candidate as a modulator of insulin signalling which acts primarily in skeletal muscle. The present experiments show that under optimal stoichometric conditions of HIR, PKC $\theta$ and IRS- 1 an almost complete inhibition on insulin receptor autophosphorylation and a strong decrease of IRS-1 phosphorylation occurs. To substantiate an inhibitory role for PKC $\theta$ on insulin signalling in skeletal muscle further experiments in skeletal tissue are necessary. Due to the low expression level of signalling elements and limited amounts of tissues which are available it will, however, be difficult to prove this effect in human skeletal muscle.

The potential mechanism by which IRS- 1 is able to mediate PKC $\theta$ dependent inhibition on the insulin receptor in human skeletal muscle could be indicated by the observation of a molecular weight shift of IRS1 in PKC $\theta$ transfected cells after phorbol ester treatment. Serine phosphorylation of IRS-1 was shown to be involved in the inhibitory effects on insulin receptor signalling [7, 8, 9]. An analogous mechanism might be important for PKC dependent receptor inhibition. Our experiments with alkaline phosphatase provide indirect evidence that a PKC $\theta$ dependent serine or threonine phosphorylation might occur and modulate insulin receptor activity. If this PKC $\theta$ dependent serine phosphorylation comprises part of the inhibitory mechanism or is a relevant marker of the inhibitory effect, it is necessary to define the phosphorylation sites on IRS-1. Since we have no direct evidence that serine phosphorylation of IRS-1 is indeed responsible for inhibition of HIR autophosphorylation, other mechanisms involving a direct complex formation between PKC and different signalling proteins have to be considered as alternative mechanisms as well.

Acknowledgements. This work was supported by the Deutsche Forschungsgemeinschaft (Ke 553/4-2). We thank M.F. White (Boston, USA) for the generous gift of $\alpha$ IRS-1 antibodies and IRS-1 cDNA.

\section{References}

1. Beck-Nielsen H, Groop LC (1994) Metabolic and genetic characterization of prediabetic states. Sequence of events leading to non-insulin-dependent diabetes mellitus. J Clin Invest 94: 1714-1721 
2. Bossenmaier B, Mosthaf L, Mischak H, Ullrich A, Häring HU (1997) Protein kinase C isoforms beta 1 and beta 2 inhibit the tyrosine kinase activity of the insulin receptor. Diabetologia 40: 863-866

3. Nishizuka Y (1988) The molecular heterogeneity of protein kinase $\mathrm{C}$ and its implications for cellular regulation. Nature 334: 661-665

4. Nishizuka Y (1992) Intracellular signalling by hydrolysis of phospholipids and activation of protein kinase C. Science 258: 607-614

5. Berti L, Mosthaf L, Kroder G et al. (1994). Glucose induced translocation of protein kinase $\mathrm{C}$ isoforms in rat-1 fibroblasts is paralleled by inhibition of the insulin receptor tyrosine kinase. J Biol Chem 269: 3381-3386.

6. Kellerer M, Mushack J, Mischak H, Häring HU (1997) Protein kinase $\mathrm{C}(\mathrm{PKC}) \varepsilon$ enhances the inhibitory effect of TNF $\alpha$ on insulin signalling in HEK 293 cells. FEBS Lett 418: $119-122$
7. Hotamisligil GS, Peraldi P, Budavari A, Ellis R, White MF, Spiegelman BM (1996) IRS-1-mediated inhibition of insulin receptor tyrosin kinase activity in TNF-alpha- and obesity-induced insulin resistance. Science 271: 665-668

8. Mothe I, VanObberghen E (1996) Phosphorylation of insulin receptor substrate 1 on multiple serine residues, 612, 632, 662, and 731, modulates insulin action. J Biol Chem 271: 11222-11227

9. De Fea K, Roth RA (1997) Protein kinase C modulation of insulin receptor substrate-1 tyrosine phosphorylation requires serine 612. Biochemistry 36: 12939-12947

10. Schmitz-Pfeiffer C, Vbrowne CL, Oakes ND et al. (1997) Alterations in the expression and cellular localization of protein kinase $\mathrm{C}$ isozymes epsilon and theta are associated with insulin resistance in skeletal muscle of the high-fatfed rat. Diabetes 46: 169-178 